Conclusions: Anti-IL-6R treatment limits proliferative ability of activated $\mathrm{CXCR}^{+} \mathrm{ICOS}^{+}$Tfh cells, blocking their emergence as well as plasmablast accumulation following influenza vaccination. Our data suggest that IL-6 is crucial for optimal in vivo generation of activated Tfh cells in humans.

Disclosure of Interest: None declared

DOI: 10.1136/annrheumdis-2018-eular.5101

\section{THU0046 SMALL MOLECULE INHIBITOR OF THE WNT PATHWAY (SM04755) AS A POTENTIAL TOPICAL TREATMENT FOR PSORIASIS}

V. Deshmukh, M. Pedraza, L. Lamangan, Y. Yazici. Samumed, LLC, San Diego, USA

Background: Psoriasis (PSO) is an autoimmune disease, causing patches of thick, inflamed, scaly skin due to excessive proliferation of skin cells ${ }^{1}$. Wht signalling plays an important role in PSO, regulating inflammation and keratinocyte pro liferation. SM04755, a novel, topical small-molecule Wnt pathway inhibitor was previously shown to inhibit inflammation and keratinocyte proliferation in vitro and in an IMQ-induced mouse PSO model ${ }^{2}$.

Objectives: In this study, the effects of SM04755 on inflammation and skin health were evaluated in two models that closely resemble human PSO pathophysiology: reconstitution of ICR scid mice with minor histocompatibility mismatched naïve CD4 ${ }^{+}$T lymphocytes ${ }^{3}$ and an IL-23 intra-dermal injection model ${ }^{4}$

Methods: For $(\mathrm{A})$ immune reconstitution model, peripheral blood mononuclear cells were isolated from $\mathrm{F}_{2}$ (BALB/c $\times 129 / \mathrm{SvJ}$ ) mice and analysed by flow cytometry to identify $\mathrm{H}-2 \mathrm{D}^{\mathrm{d}}$ haplotype donor mice. $\mathrm{CD} 4^{+} / \mathrm{CD} 45 \mathrm{RB}{ }^{\mathrm{Hi}}$ cells from donor mice spleens were purified and injected intravenously into CB17/ICR-Tac Prkdc/ scid (ICR scid) mice $\left(5 \times 10^{5}\right.$ cells/mouse). Skin appearance and ear thickness were evaluated weekly. At the first visible PSO-like signs, mice were randomised and treated with SM04755 $\left(400 \mu \mathrm{g} / \mathrm{cm}^{2}\right)$ or vehicle. After 14 weeks, body and spleen weights were measured, and inflammation was evaluated by measuring cytokines (IL-1 $\beta$, TNF- $\alpha$, IL-6) in tissues from skin, ears, spleen and plasma using ELISA. Epidermal thickness and skin immune cell infiltration were histologically evaluated. For (B) the IL-23 model, rIL-23 was injected intra-dermally into mouse ears, every other day for 35 days. Mice were randomised on Day 16 and treated with SM04755 $\left(400 \mu \mathrm{g} / \mathrm{cm}^{2}\right)$ or vehicle or Clobetasol daily for 20 days. Ear thickness was measured every 3 days. Skin immune cell infiltration was histologically evaluated

Results: (A) Immune reconstitution of ICR scid mice resulted in PSO-like signs, with skin lesions and increased thickness of the skin and ears. Treatment with topical SM04755 $\left(400 \mu \mathrm{g} / \mathrm{cm}^{2}\right)$ significantly $(p<0.01)$ decreased skin and ear thicknesses and improved skin appearance compared to vehicle. Body weights were significantly $(p<0.05)$ higher in treated compared to vehicle mice. SM04755 significantly reduced histologically measured epidermal thickness $(p<0.05)$ and immune cell infiltration in the skin compared to vehicle. Further, inflammatory cytokine levels in the skin, ears, spleen and plasma and spleen weight were significantly $(p<0.05)$ reduced in SM04755 treated animals compared with vehicle. $(B)$ Intradermal IL-23 injection into mouse ears resulted in inflammation and ear thickening by day 16 compared to sham. Treatment with topical SM04755 $\left(400 \mu \mathrm{g} / \mathrm{cm}^{2}\right) \mathrm{sig}$ nificantly $(p<0.05)$ decreased ear thickness, immune cell infiltration, and improved appearance compared to vehicle.

Conclusions: In two mouse models of $(A)$ minor histocompatibility mismatched $T$ lymphocyte reconstitution-induced PSO and (B) IL-23 injection-induced PSO, topically applied SM04755 inhibited key pathophysiological features of PSO at macro- and microscopic levels, compared to vehicle. SM04755 has potential as a topical therapy for PSO. Clinical trials are ongoing.

\section{REFERENCES:}

[1] National PSO Foundation.

[2] Deshmukh, et al. Arthritis Rheumatol. 2016;68(suppl 10).

[3] Schon, et al. Nature Medicine, 1997 Feb.

[4] Mabuchi et al. J Immunol. 2011 Nov 15;187(10).

Disclosure of Interest: V. Deshmukh Shareholder of: Samumed, LLC, Employee of: Samumed, LLC, M. Pedraza Shareholder of: Samumed, LLC, Employee of: Samumed, LLC, L. Lamangan Shareholder of: Samumed, LLC, Employee of: Samumed, LLC, Y. Yazici Shareholder of: Samumed, LLC, Employee of: Samumed, LLC

DOI: 10.1136/annrheumdis-2018-eular.4752

\section{THU0047 \\ 1,25(OH)2D3 AND DEXAMETHASONE ADDITIVELY SUPPRESS SYNOVIAL FIBROBLAST ACTIVATION BY CCR6+ TH MEMORY CELLS AND ENHANCE THE EFFECT OF TNF-ALPHA BLOCKADE}

W. Dankers ${ }^{1,2}$, W. Dankers ${ }^{1,2}$, N. Davelaar ${ }^{1,2}$, P.S. Asmawidjaja ${ }^{1,2}$, A.M. Mus ${ }^{1,2}, \mathrm{~J}$ M. Hazes ${ }^{1}$, E.M. Colin ${ }^{3}$, E. Lubberts ${ }^{1,2}$. ${ }^{1}$ Rheumatology; ${ }^{2}$ Immunology, Erasmus MC, Rotterdam; ${ }^{3}$ Rheumatology, Hospital Group Twente, Almelo, Netherlands

Background: Despite improvement in treatment of rheumatoid arthritis (RA) over the past decades, insufficient treatment response and treatment resistance in many patients demonstrate the need to develop new therapeutic strategies. Chronic synovial inflammation could be suppressed by targeting activation of RA synovial fibroblasts (RASF) by for example IL-17A-producing CCR6 +T helper memory (memTh) cells. Previously, we have shown that dexamethasone (DEX) combined with the active vitamin $\mathrm{D}$ metabolite $1,25(\mathrm{OH})_{2} \mathrm{D}_{3}$ reduces pathogenicity of memTh cells.

Objectives: To study the additive effect of $1,25(\mathrm{OH})_{2} \mathrm{D}_{3}$ and DEX on suppressing the pro-inflammatory loop between RASF and CCR6 +memTh cells and explore potential therapeutic applications.

Methods: CCR6 +memTh cells from PBMC of healthy donors or treatment-naïve early RA patients were cultured alone or with RASF from established RA patients for three days and treated with or without $1,25(\mathrm{OH})_{2} \mathrm{D}_{3}$, DEX or etanercept. Treatment effects were assessed using ELISA and flow cytometry.

Results: CCR6 +memTh produces less of the pro-inflammatory cytokines IL17A, IL-22 and IFN $\gamma$ upon exposure to $1,25(\mathrm{OH})_{2} \mathrm{D}_{3}$, and to a lesser extent by DEX. TNF $\alpha$ was only inhibited by the combination of $1,25(\mathrm{OH})_{2} \mathrm{D}_{3}$ and DEX. In contrast, in RASF cultures DEX was the strongest inhibitor of IL-6, IL-8 and tissuedestructive enzymes. As a result, $1,25(\mathrm{OH})_{2} \mathrm{D}_{3}$ and $\mathrm{DEX}$ additively inhibited inflammatory mediators in CCR6 +memTh RASF co-cultures. Interestingly, low doses of mainly DEX, but also $1,25(\mathrm{OH})_{2} \mathrm{D}_{3}$, combined with etanercept better suppressed synovial inflammation in this co-culture model compared to etanercept alone.

Conclusions: This study suggests that $1,25(\mathrm{OH})_{2} \mathrm{D}_{3}$ and $\mathrm{DEX}$ additively inhibit synovial inflammation through targeting different pro-inflammatory mechanisms. Furthermore, low doses of DEX and $1,25(\mathrm{OH})_{2} \mathrm{D}_{3}$ enhance the effect of TNF $\alpha$ blockade in inhibiting RASF activation, providing a basis to improve RA treatment. Disclosure of Interest: None declared DOI: 10.1136/annrheumdis-2018-eular.6973

\section{THU0048 PRO-INFLAMMATORY IL-17A-PRODUCING CCR6+ T HELPER MEMORY CELLS CHANGE INTO ANTI- INFLAMMATORY CELLS WITH REGULATORY CAPACITY UPON EXPOSURE TO ACTIVE VITAMIN D}

W. Dankers ${ }^{1,2}$, N. Davelaar ${ }^{1,2}$, J.P. van Hamburg ${ }^{1,2}$, J. van de Peppel ${ }^{3}$, E. M. Colin ${ }^{4}$, E. Lubberts ${ }^{1,2} .{ }^{1}$ Rheumatology; ${ }^{2}$ Immunology; ${ }^{3}$ Internal Medicine, Erasmus MC, Rotterdam; ${ }^{4}$ Rheumatology, Hospital Group Twente, Almelo, Netherlands

Background: In autoimmune diseases such as rheumatoid arthritis (RA), an important therapeutic goal is to normalise the imbalance between pro- and antiinflammatory cells. In RA, especially pro-inflammatory CCR6 +T helper (Th) memory cells, characterised by IL-17A production and RORC expression, are elevated and more activated compared to healthy controls. Therefore, modulating these cells to become anti-inflammatory could contribute to restoring the immunological balance. Interestingly, the active vitamin $\mathrm{D}$ metabolite $1,25(\mathrm{OH})_{2} \mathrm{D}_{3}$ inhibits pro-inflammatory cytokine production by CCR $6+$ Th memory cells.

Objectives: We investigated whether $1,25(\mathrm{OH})_{2} \mathrm{D}_{3}$ can induce an anti-inflammatory phenotype in these memory CCR 6 +Th cells.

Methods: CCR6 +Th memory cells, excluding Tregs, were sorted from treatment-naïve early RA patients or healthy controls and cultured with or without 1,25 $(\mathrm{OH})_{2} \mathrm{D}_{3}$. Effects were analysed using microarray, RT-PCR, ELISA or flow cytometry. Functional properties were assessed via suppression and chemotaxis assays.

Results: $1,25(\mathrm{OH})_{2} \mathrm{D}_{3}$ inhibits pro-inflammatory cytokines such as IL-17A, IL-17F and IL-22 in CCR 6 +Th memory cells from both healthy controls and RA patients This is accompanied by induction of anti-inflammatory factors, including IL-10 and CTLA4. Interestingly, these formerly pathogenic cells suppress proliferation of autologous CD3 + T cells, similar to classical Tregs. Importantly, the modulated memory cells still migrate towards the site of inflammation, modelled by synovial fluid, and retain their suppressive capacity in this environment. 
Conclusions: Committed pro-inflammatory IL-17A-producing CCR6 +Th memory cells shift towards anti-inflammatory cells with functional regulatory capacities upon exposure to active vitamin $\mathrm{D}$. This process can contribute to restoring the immunological balance and inhibiting synovial inflammation in RA. Disclosure of Interest: None declared

DOI: 10.1136/annrheumdis-2018-eular.6997

\section{THU0049 DEVELOPMENT OF TFH-TH1 LIKE CELLS THROUGH EPIGENETIC MODIFICATION BY STATS FAMILY FACTORS IN PATIENTS WITH SYSTEMIC LUPUS ERYTHEMATOSUS}

$\underline{\text { X. Ma }}{ }^{1,2}$, S. Nakayamada ${ }^{1}$, S. Kubo ${ }^{1}$, K. Sakata ${ }^{1,3}$, K. Yamagata ${ }^{1}$, Y. Tanaka ${ }^{1}$ ${ }^{1}$ First Department of Internal Medicine, School of Medicine, University of Occupational and Environmental Health, Japan, Kitakyushu, Japan; ${ }^{2}$ Department of Pediatrics, The First Hospital of China Medical University, Shenyang, China: ${ }^{3}$ Mitsubishi Tanabe Pharma, Yokohama, Japan

Background: Systemic lupus erythematosus (SLE) is a prototype of autoimmune disease characterised by chronic immune activation and multiple immunologic phenotypes $^{(1)}$. Among several types of immune cells, T follicular helper (Tfh) cells serve important roles in the development and progression of $\mathrm{SLE}^{(2)}$.

Objectives: To assess the characteristics and mechanisms of differentiation of Tfh cells, we probed the phenotype of T helper cells in patients with SLE and underlying epigenetic modifications by cytokine-induced signal transducer and activators of transcription (STAT) family factors.

Methods: Naive $\mathrm{CD} 4^{+} \mathrm{T}$ cells and memory $\mathrm{CD} 4^{+} \mathrm{T}$ cells were isolated and stimulated by various cytokines and T cell receptor (TCR) in vitro. Expression of characteristic markers of Tfh-Th1-cells and phosphorylation of STATs were analysed by flow cytometry and qPCR. Histone modifications were evaluated by chromatin immunoprecipitation. Peripheral blood mononuclear cells from SLE patients and healthy controls were analysed by flow cytometry and productions of cytokines in serum were tested by cytometric bead array.

Results: Differentiation of CXCR5 ${ }^{+} \mathrm{CXCR} 3^{+} \mathrm{Bcl}-6^{+}{ }^{\mathrm{T}}-\mathrm{bet}^{+} \mathrm{IL}-21^{+} \mathrm{IFN}-\gamma^{+}$Tfh-Th1like cells was induced by IL-12. Among STAT family, STAT1 and STAT4 were phosphorylated simultaneously by IL-12 independent of IFN- $\gamma$ and directly bound on Bcl-6 and T-bet gene loci accompanied by suppression of trimethylated histone 3 lysine 27. Compared with healthy controls, responsiveness of activation of STAT1 and STAT4 by IL-12 and proportion of activated Tfh-Th1-like cells were increased in patients with SLE.

Conclusions: Our findings suggest that IL-12-mediated co-activation of STAT1 and STAT4 alter histone modification, resulting in development of Tfh-Th1-like cells that are characteristically expanded in patients with SLE. These findings could be one of underlying pathogenesis of SLE and potentially helpful towards development of cell-specific treatment.

\section{REFERENCES:}

[1] Tsokos GC. Systemic lupus erythematosus. N Engl J Med. 2011;365 (22):2110-21.

[2] Suarez-Fueyo A, et al. T cells in systemic lupus erythematosus. Curr Opin Immunol 2016;43:32-8.

Acknowledgements: The authors thank Ms. N. Sakaguchi for the excellent technical assistance.

Disclosure of Interest: None declared

DOI: 10.1136/annrheumdis-2018-eular.1441

THURSDAY, 14 JUNE 2018

\section{Rheumatoid arthritis - etiology, pathogenesis and animal models}

\section{THU0050 \\ HYPOXIA INDUCES PRODUCTION OF CITRULLINATED PROTEINS IN HUMAN FIBROBLAST-LIKE SYNOVIOCYTES THROUGH REGULATING HIF1A}

R. Yu, C. Li, L. Sun, L. Jian, Z. Ma, J. Zhao, X. Liu. Department of Rheumatology and Immunology, Peking University Third Hospital, Beijing, China

Background: Hypoxia is a prominent microenvironment feature in a range of disorders including cancer, rheumatoid arthritis (RA), atherosclerosis, inflammatory bowel disease (IBD), infection and obesity. Hypoxia promotes biological functions of fibroblast-like synoviocytes via regulating hypoxia-inducible factor $1 \alpha(\mathrm{HIF} 1 \alpha)$. Dysregulated protein citrullination in RA drives the production of antibodies to citrullinated proteins, a highly specific biomarker of RA. However, the mechanisms promoting citrullination in RA are not yet fully elucidated.
Objectives: In the present study, we investigated whether pathophysiological hypoxia as found in the rheumatoid synovium modulates the citrullination in human fibroblast-like synoviocytes (HFLS).

Methods: HFLS were incubated in a hypoxic chamber $\left(5 \% \mathrm{CO}_{2}\right.$ and $1 \% \mathrm{O}_{2}$, balanced with $\mathrm{N}_{2}$ as indicated) or in a normal incubator containing $5 \% \mathrm{CO}_{2}$ and approximately $20 \% \mathrm{O}_{2}$. The realtime quantitative PCR and western blotting were used to detect the expression of peptidylarginine deiminase 2, peptidylarginine deiminase 4 and citrullinated proteins. HFLS were transfected with HIF1 $\alpha$ siRNA to block the HIF1 $\alpha$ pathway.

Results: In the present study, we found that peptidylarginine deiminase 2 (PAD2) and citrullinated proteins were increased in HFLS after exposed to hypoxia. Moreover, knocking down HIF1 $\alpha$ by hif $1 \alpha$ siRNA ameliorated the expression of PAD2 and citrullinated proteins.

Conclusions: Collectively, the present study provide a new mechanism involved in generating citrullinated proteins: hypoxia promote citrullination and PAD production in HFLS. Concurrently, we also proposed a novel hypoxia involved mechanism in RA pathogenesis. The present study deepens our understanding of the role of hypoxia in the pathogenesis of RA and provides potential therapeutic strategy for RA.

Acknowledgements: We thank Jiansheng Huang for providing language help and revising the manuscript.

Disclosure of Interest: None declared

DOI: 10.1136/annrheumdis-2018-eular.6626

\section{THU0051 TNF RECEPTOR 2 PLAYS AN IMMUNOREGULATORY AND ANTI-INFLAMMATORY ROLE IN ARTHRITIS}

W.-Y. Tseng ${ }^{1,2}$, I.-S. Huang ${ }^{1}$, F. Clanchy ${ }^{1}$, K. Mcnamee ${ }^{1}$, F. Mccann ${ }^{1}$, R. O. Williams ${ }^{1}$. ${ }^{1}$ The Kennedy Institute of Rheumatology, University of Oxford, Oxford, UK; ${ }^{2}$ Division of Rheumatology, Allergy and Immunology, Chang Gung Memorial Hospital at Keelung, Keelung, Taiwan, Province of China

Background: Despite the overall success of TNF $\alpha$ inhibitors in rheumatoid arthritis (RA), up to half of patients are classified as either primary or secondary nonresponders ${ }^{1}$. One hypothesis put forward to explain resistance to anti-TNF $\alpha$ therapy is an ascendant effect of dysregulated regulatory T cells and increased Th17 responses following TNF $\alpha$ blockade. Previous studies have demonstrated that TNFR2 is critical for stabilisation and suppressive function of regulatory T cells ${ }^{2,3}$ However, TNFR2 also activates pro-inflammatory signalling cascades and, to date, the net effect of TNFR2 on the pathogenesis of RA remains unclear.

Objectives: In this study we address this question by assessing the progression of collagen-induced arthritis (CIA) in mice deficient for TNFR1 or TNFR2.

Methods: C57BI/6N.Q (H-2 $\left.{ }^{q}\right)$ mice were immunised with bovine type II collagen emulsified in complete Freund's adjuvant. The mice were monitored daily for arthritis and scored clinically from the day of onset of disease. Mice were culled on day 10 after arthritis onset and spleens, lymph nodes, serum and paws were collected for further analysis.

Results: As expected, TNFR $1^{-/-}$mice were found to be largely resistant to arthritis both clinically and histologically (figure 1). In contrast, there was significantly enhanced disease activity at the clinical and histological levels in TNFR2 ${ }^{-1-}$ mice (figure 1) and this was accompanied by increased expression of the pro-inflammatory cytokines, TNF $\alpha$ and IL-6, reduced numbers of regulatory T cells, reduced FoxP3 expression and reduced expression of the immune inhibitory molecules, $\mathrm{PD}-1$ and LAG3, in TNFR2 ${ }^{-1-}$ mice compared to WT mice.

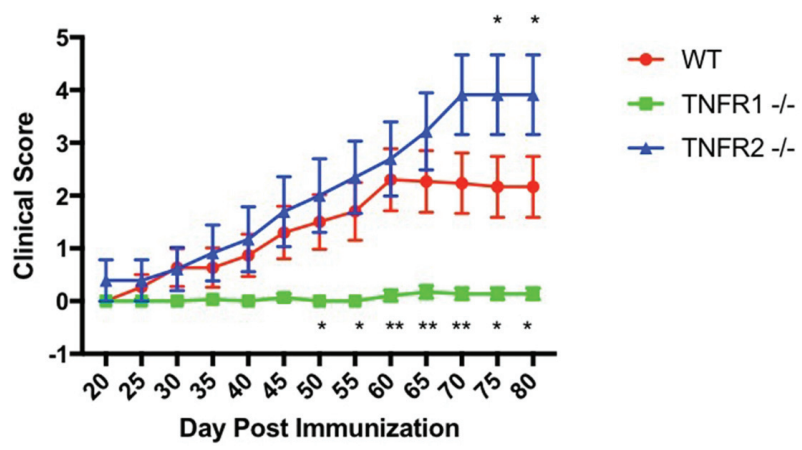

Abstract THU0051 - Figure 1. Progression of CIA in mice deficient for TNFR1 or TNFR2

Conclusions: This study has shown that TNFR2 signalling plays immunoregulatory and anti-inflammatory roles in CIA. First, it contributes to promotion of regulatory T cell generation and FoxP3 expression, and second, it limits the expression of pro inflammatory cytokines. TNFR2 also regulates the expression immune inhibitory 